\title{
A nonlinear model of ionic wave propagation along microtubules
}

\author{
M. V. Satarić • D. I. Ilić • N. Ralević $\cdot$ \\ Jack Adam Tuszynski
}

Received: 3 September 2009 / Published online: 17 September 2009

(C) European Biophysical Societies' Association 2009

\section{Erratum to: Eur Biophys J (2009) 38:637-647 DOI 10.1007/s00249-009-0421-5}

In our recent paper (Satarić et al. 2009), the Introduction section contained statements regarding the $C$-terminal tails that have inadvertently been included without properly citing the source of this information provided. In particular, the passage given below should be attributed to Georgiev et al. (2004) who almost 5 years earlier correctly pointed out the importance of the $C$-terminal tail sequence and structure. "Each tubulin monomer of the MT lattice has a short $C$-terminal helix $\mathrm{H} 12$ followed by a highly acidic amino acid sequence projecting out of the MT outer surface called a tubulin tail (TT). The TTs are short polypeptide sequences of 4-5 nm length when completely outstretched, protruding from the MT surface into the solution. They are essential for MT interactions with MT associated proteins and motor proteins.

Jimenez et al. (1999) determined the helicity of a (404-451) and b (394-445) tubulin $C$-terminal recombinant peptides with the use of NMR spectroscopy. The $\alpha$-tubulin TT

The online version of the original article can be found under doi:10.1007/s00249-009-0421-5.

M. V. Satarić · D. I. Ilić $(\bowtie) \cdot$ N. Ralević

Faculty of Technical Sciences, University of Novi Sad,

Trg D. Obradovića 6, 21000 Novi Sad, Serbia

e-mail: idilic@EUnet.yu

J. A. Tuszynski

Division of Experimental Oncology, Cross Cancer Institute, 11560 University Avenue, Edmonton, AB T6G 1Z2, Canada e-mail: jtus@phys.ualberta.ca amino acid sequence is EEVGADSVEGEGEEEGEEY. The $\beta$-tubulin tail is 19 amino acids long and possesses 10 negatively charged residues. The situation in the tubulin $C$-terminal domain is even more interesting. Jimenez et al. found a helix there, which is 9 amino acids longer than in tubulin. This suggests an extension into the protein, supporting the possibility of a functional coil-to-helix transition at the TT zone. The $\beta$-tubulin TT helix H12 is formed by amino acid residues (408-431) but it seems that the reversible transition between coil and the helix comprising the last 9 amino acid residues (423-431) from $\beta$-tubulin TT helix (with sequence QQYQDATAD) could either increase or decrease the length of $\mathrm{H} 12$, at the same time changing the $\beta$-TT length.

The corresponding residues (432-445) contain the 14 amino acids EQGEFEEEEGEDEA with 9 negatively charged residues $(\mathrm{E}, \mathrm{A})$ ".

The omission of the proper reference and citation has not been intentional on our part but we deeply regret not having given proper care and attention to the source of the statement and wish to apologize to the authors for it.

\section{References}

Georgiev D, Papaioanou S, Glazebrook J (2004) Neuronic system inside neurons: molecular biology and biophysics of neuronal microtubules. Biomed Rev 15:67-75

Jimenez MA, Evangelio JA, Aranda C, Lopez-Brauet A et al (1999) Helicity of alpha (404-451) and beta (394-445) tubulin $C$-terminal recombinant peptides. Protein Sci 8:788-799

Satarić M, Ilić D, Ralević N, Tuszynski J (2009) A nonlinear model of ionic wave propagation along microtubules. Eur Biophys J 38:637-647 